\title{
Dynamic Analysis of Line Gear Pair Based on Numerical Manifold Method
}

\author{
Jiang Ding $1,2,{ }^{*}$ - Aiping Deng 1 - Liwei Liu ${ }^{1}$ - Mengen Lu ${ }^{1}$ \\ ${ }^{1}$ Guangxi University, College of Mechanical Engineering, China \\ 2Guangxi University, Guangxi Key Laboratory of Manufacturing System \& Advanced Manufacturing Technology, China
}

\begin{abstract}
As a kind of tiny gear based on space curve meshing theory, the line gear is very suitable for miniaturized machines due to its compact size and low weight. However, the line gear usually suffers from serious vibration problems since its line teeth are designed as twisted threedimensional cantilevers to provide conjugated meshing curves. A dynamic model of the line gear pair is established in this paper using the numerical manifold method (NMM) to alleviate its vibration conditions, which can simultaneously provide mathematical and physical covers. The displacement function is first derived for the line teeth, and the dynamic equations of the manifold element are acquired. After inspecting the reasons that cause meshing excitation, the dynamic response of the line teeth is attained in all three orthogonal directions. The attained dynamic response shows that the vibration in the axial gear direction is more significant than that in the curvature direction. Furthermore, the vibration differential equations of the line teeth are solved through a detailed example, and the relationship between the design parameters and the natural frequency is revealed. The vibration characteristics of the first four order of the line gear are revealed through the method of NMM and compared with the result that is carried out through the commercial finite element method (FEM). The comparison shows that NMM can efficiently relieve the vibration problems of the line gear.
\end{abstract}

Keywords: line gear, dynamic response, vibration, numerical manifold method

Highlights

- The line gear is designed to be applied in miniaturized machines.

- The line teeth of the line gear are twisted three-dimensional cantilevers.

- A dynamic model of the line gear is established through the numerical manifold method.

- The numerical manifold method provides mathematical and physical covers simultaneously.

\section{INTRODUCTION}

As core parts to transmit motion and moment in miniaturized machines, tiny gear-boxes and the minigears inside them have raised significant concern. These days, many new gears have been proposed [1] to [3]. Among them, the line gear, as a tiny gear based on space curve meshing theory, has shown great potential in precise transmission in limited space. The line gear has been used in several kinds of small gear transmissions [4], but its line teeth usually suffer from fatal vibration problems and meshing transmission failure during the meshing process [5].

Many traditional gears also face similar vibration problems. Yi Yang et al. [6] investigated the non-linear dynamic response of a spur gear based on periodic mesh stiffness to improve the dynamic characteristics, and Zong Meng et al. [7] studied the vibration response and analysed fault characteristics of gears. To alleviate the vibration conditions, Belingardi et al. [8] made a dynamic analysis of a gear transmission system for an electric vehicle through a multibody approach, and Marco Cirelli et al. [9] presented a novel implementation of a specific multibody model through the tip relief micro-modification on spur gears. In addition, the method of simulating gear pair dynamic response is a potential research direction. Cirelli et al. [10] proposed a refined methodology to simulate the non-linear dynamic response of spur gears through the multibody model based on a penalty contact formulation and considers teeth. Ebrahimi and Eberhard [11] established a rigid-elastic modelling of meshing gear wheels to investigate the effects of multi-tooth contact, as well as backlash and left and right-hand side contact of the meshing teeth. The calculation of gear meshing stiffness is indispensable in the dynamic analysis of gear pairs, and is also one of the research directions for scholars. Cooley et al. [12] studied the calculation methods of gear meshing stiffness, and compared the average slope method and local slope method. Luo et al. [13] proposed a tooth tip modelling method based on defect ratio and independent of gear shape to better calculate the timevarying meshing stiffness of the gear. However, due to the irregular shape of the line teeth, the dynamic study on the traditional gears cannot be applied to alleviate the vibration conditions of the line gear [5].

As shown in Fig. 1, the line teeth can be considered multiple twisted three-dimensional space curved cantilever beams axisymmetrically fixed 
around the end of the wheel cylinder. The vibration problems of the line gear are mainly caused by these cantilever beams [5]. For now, many theoretical models of three-dimensional cantilever beams have been studied. Wang and Li [14] calculated the natural frequency of functionally graded cantilever beam and analysed the effects of material gradient parameters, the length-depth ratio of cantilever beam and boundary conditions on the vibration. Zhang et al. [15] investigated the natural frequency and mode of pyramidal Timoshenko beams, and analysed the effects of mechanical properties, rotational inertia and shear deformation on the natural frequency of beams with different taper ratios. Zhao and $\mathrm{Wu}$ [16] established the motion coupling equation of a rotating three-dimensional cantilever beam, and showed the effects of Coriolis term and steady-state axial deformation on coupling vibration. It is worth noting that stress and deformation analysis of the cantilever beam is an important research direction. For instance, Zhou et al. [17] investigated the 3D dynamics of a rotary functional gradient cantilever beam and revealed the couplings among the axial, flap-wise, and chordwise deformations. However, the previous models mainly focus on a 3D cantilever of normal shapes, rather than twisted three-dimensional space curved cantilever beams in the line gears.

In this paper, we propose the numerical manifold method (NMM) to relieve the dynamic problems of the line gear. The NMM is a numerical calculation method based on the concept of manifold elements, and has been widely used in solving continuous linear elasticity problems [18], crack problems [19], and continuous stress-strain field problems [20]. The research results show that NMM can improve computational accuracy and convergence compared with the finite element method [21]. Wei-bin WEN et al. [22] presented a new NMM based on quarticuniform B-spline interpolation, which has high interpolation accuracy and rapid convergence. Compared to other methods, the NMM can provide mathematical covers and physical covers simultaneously. The mathematical cover is not limited by the physical cover since unknown variables contained in mathematical covers are no longer corresponding to the node displacement. Therefore, the NMM can reduce the sensitivity of unit distortion in the line gears and efficiently obtain numerical results with decent accuracy.

In this paper, the dynamic equations of manifold element are established through the NMM, and the vibration differential equation of the cylindrical helical line tooth is derived from the stress analysis of the line tooth micro-element. The effects of the main design parameters of line gears on their natural frequency are analysed. The dynamic response of the line gear pair and the natural vibration modes of the first four orders are revealed, and the comparison between the natural frequency result computed with the commercial finite element method (FEM) and the NMM is carried out to verify further that the dynamic model established by the NMM.

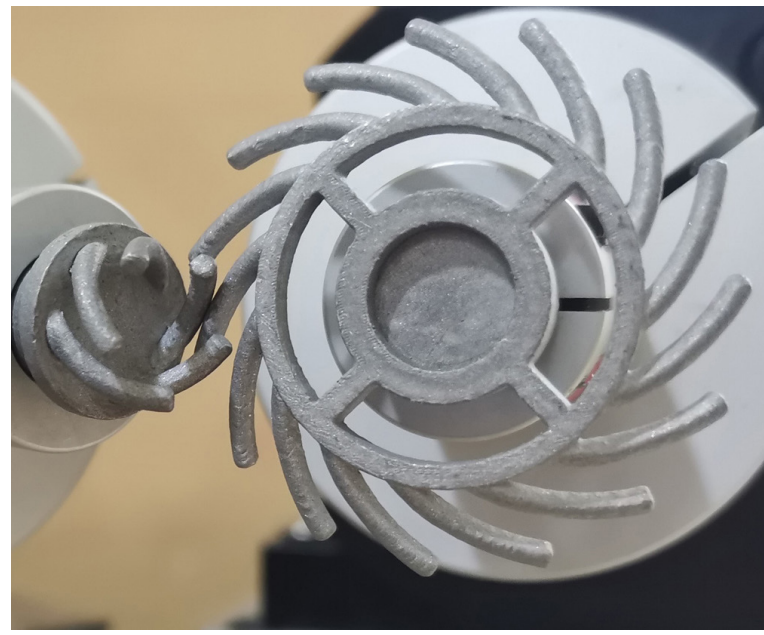

Fig. 1. Space curved cantilever beams on a pair of line gears

\section{DYNAMIC MODEL}

The usual interpolation functions for NMM include polynomial function, trigonometric function, and B-spline function. Polynomials and trigonometric functions are widely used due to their convenient calculation, but they are inaccurate and too sensitive for the mesh distortion when describing the displacement continuity in a pair of line gears. In contrast, the B-spline interpolation function possesses advantages of continuity, local support, local control and modification, and can be employed to solve mechanical problems effectively [23]. Therefore, the B-spline function is adopted as the interpolation function of NMM in this paper for the line teeth, which are sensitive to the unit deformation.

\subsection{Displacement Function of Manifold Element}

The line gear meshing model and the meshing coordinate systems of line gears are shown in Fig. 2. The driving gear is a cylindrical helical line gear. The rotating speed of driving gear is donated as $\omega_{1}$, and the rotating speed of driven gear as $\omega_{2} . o_{1}-x_{1} y_{1} z_{1}$ and $o_{1}^{\prime}-x_{1}^{\prime} y_{1}^{\prime} z_{1}^{\prime}$ are the fixed coordinate system and the rotating coordinate system of the driving gear, respectively. $o_{2}-x_{2} y_{2} z_{2}$ and $o_{2}^{\prime}-x_{2}^{\prime} y_{2}^{\prime} z_{2}^{\prime}$ are the fixed 
coordinate system and the rotating coordinate system of the driven gear, respectively. The angle of the centre axis is $(\pi-\theta)$. The pair of interaction meshing forces between the driving gear and the driven gear are donated as $F$ and $F^{\prime}$, respectively.

a)

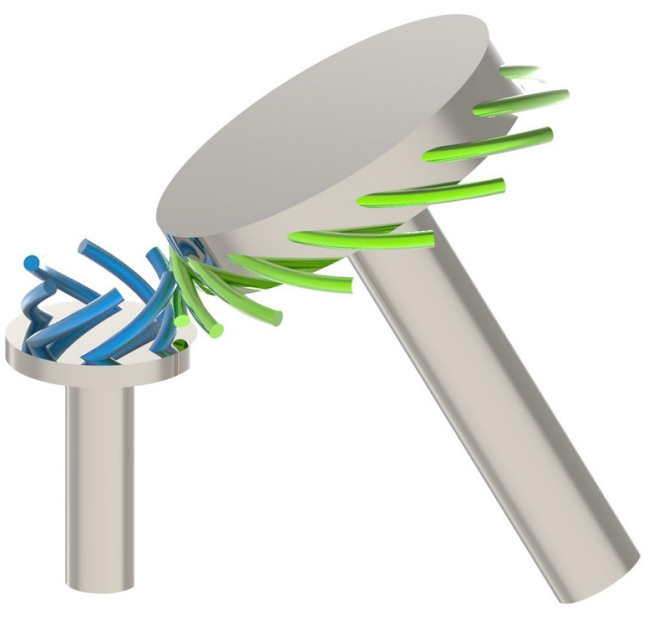

b)

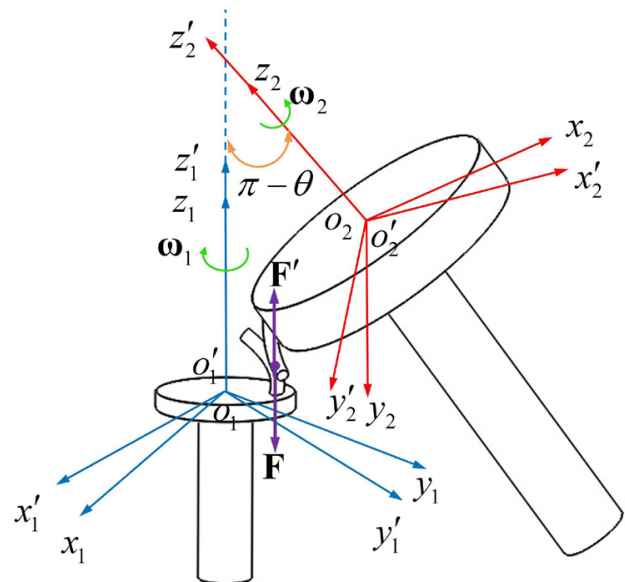

Fig. 2. Simulation diagrams of line gears: a) meshing model; b) meshing coordinate systems

The B-spline interpolation function has different kinds of definitions in mathematics. To ensure the coming programming practical, the B-spline basis function in this paper is explicitly defined by recursion and expressed in polynomial form. The B-spline basis function is defined as:

$$
\left\{\begin{array}{rl}
B_{i, 0}(x)= & \begin{cases}1 & x_{i^{\prime}} \leq x_{i^{\prime}+1} \\
0 & \text { others }\end{cases} \\
B_{i, k}(x) & =\frac{x-x_{i}}{x_{i+k}-x_{i}} B_{i, k-1}(x) \\
& +\frac{x_{i+k+1}-x}{x_{i+k+1}-x_{i+1}} B_{i+1, k-1}(x)
\end{array},\right.
$$

where $\left[x_{i^{\prime}}, x_{i^{\prime}+1}\right]$ is defined as the B-spline node interval $i^{\prime}, i$ is the number of B-spline, and $k$ is the order of B-spline function.

To mesh the line gear model, the threedimensional B-spline interpolation function needs to be established. Through Eq. (1), B-spline functions in $x, y$ and $z$ directions can be obtained, respectively. Three-dimensional B-spline interpolation functions can be obtained as follows:

$$
\begin{aligned}
N_{e} & =\sum_{n_{1}=0}^{k_{1}} \sum_{n_{2}=0}^{k_{2}=0} \sum_{n_{3}=0}^{k_{3}} B_{i-k_{1}+n_{1}, k_{1}}(x) B_{j-k_{2}+n_{2}, k_{2}}(y) B_{l-k_{3}+n_{3}, k_{3}}(z) \\
& =1,
\end{aligned}
$$

where $k_{1}, k_{2}$ and $k_{3}$ are the order of B-spline function in $x, y$ and $z$ directions respectively, and $i, j$ and $l$ are the number of B-spline in $x, y$ and $z$ directions respectively.

Eq. (2) indicates that the three-dimensional B-spline function satisfies the requirement of weight function; thus, the B-spine function can be taken as the covering weight function of the threedimensional manifold element. The $k$ order B-spline polynomial function with non-repeated nodes has $k-1$ order continuity and coordination in the whole element, which ensures the solution accuracy. The B-spline basis function has high order continuity and coordination, and obtains decent interpolation precision. Each weight function should correspond to the local mathematical cover $U_{i-k_{1}+n_{1}, j-k_{2}+n_{2}, l-k_{3}+n_{3}}$, and each manifold element corresponds to at least one local cover function. The whole displacement function of manifold element $e$ is:

$$
\mathbf{U}^{(e)}=\mathbf{N}_{e} \times\left(\mathbf{S D}^{(e)}\right)=\mathbf{T}^{(e)} \mathbf{D}^{(e)},
$$

where $\mathbf{N}_{e}, \mathbf{S}, \mathbf{T}^{(e)}$ and $\mathbf{D}^{(e)}$ are the interpolation function matrix of the element $e$, the order matrix of the local cover function, the covering matrix of the element $e$ and the degree of freedom matrix of the element $e$, respectively. Also, matrix $\mathbf{D}^{(e)}$ contains time variable, $t$. $\mathbf{T}^{(e)}$ and $\mathbf{D}^{(e)}$ are expressed as:

$$
\begin{aligned}
\mathbf{T}^{(e)} & =\left[\begin{array}{llll}
\mathbf{N}_{1} \mathbf{S} & \mathbf{N}_{2} \mathbf{S} & \cdots & \mathbf{N}_{n} \mathbf{S}
\end{array}\right], \\
\mathbf{D}^{(e)} & =\left[\begin{array}{lll}
d_{i-k_{1}, j-k_{2}, l-k_{3}} & \cdots & d_{i, j, l}
\end{array}\right]^{T} .
\end{aligned}
$$

The cover form of element $e$ is shown in Fig. 3. The overlap part of each local cover function and the physical domain is manifold element $e$. The global cover function on the solution domain can be obtained through the cover function and weight function on each manifold element. 


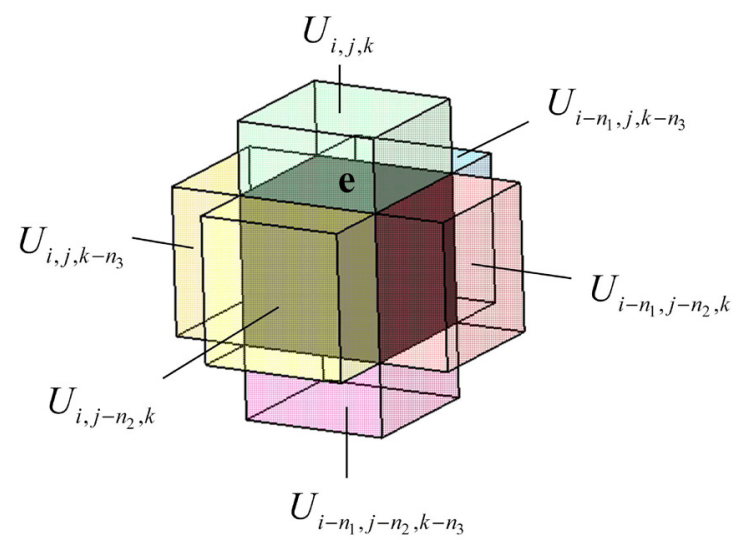

Fig. 3. Relation between manifold element and local mathematical cover

\subsection{Dynamic Equation of Manifold Element}

The vibration characteristic of the manifold element $e$ is investigated without loss of generality. The linear elastic strain energy matrix $\mathbf{V}^{e}$ is:

$$
\mathbf{V}^{e}=\frac{1}{2} \iiint_{\Omega}\left[\varepsilon^{e}\right]^{T} \sigma^{e} \mathrm{~d} x \mathrm{~d} y \mathrm{~d} z,
$$

where $\boldsymbol{\sigma}^{e}$ and $\boldsymbol{\varepsilon}^{e}$ are the stress matrix and strain matrix of the manifold element $e$, respectively. $\Omega$ is the integral domain. $\boldsymbol{\sigma}^{e}$ and $\boldsymbol{\varepsilon}^{e}$ are expressed as:

$$
\begin{gathered}
\boldsymbol{\varepsilon}^{e}=\mathbf{Q} \mathbf{U}^{(e)}=\mathbf{Q} \mathbf{T}^{(e)} \mathbf{D}^{(e)}=\mathbf{B}^{e} \mathbf{D}^{(e)}, \\
\boldsymbol{\sigma}^{e}=\mathbf{E} \boldsymbol{\varepsilon}^{e}=\mathbf{E} \mathbf{Q} \mathbf{T}^{(e)} \mathbf{D}^{(e)}=\mathbf{E} \mathbf{B}^{e} \mathbf{D}^{(e)},
\end{gathered}
$$

where $\mathbf{Q}, \mathbf{E}$ and $\mathbf{B}$ are the differential operator matrix, the elastic matrix and the element strain transformation matrix, respectively. The strain energy matrix $\mathbf{V}^{e}$ and kinetic energy matrix $\mathbf{W}^{e}$ of the manifold element $e$ can be obtained as follows:

$$
\begin{array}{r}
\mathbf{V}^{e}=\frac{1}{2}\left[\mathbf{D}^{(e)}\right]^{T} \iiint_{\Omega}\left[\mathbf{B}^{e}\right]^{T} \mathbf{E} \mathbf{B}^{e} \mathrm{~d} x \mathrm{~d} y \mathrm{~d} z \cdot \mathbf{D}^{(e)}, \\
\mathbf{W}^{e}=\frac{1}{2} \iiint_{\Omega} \rho\left[\frac{\partial \mathbf{U}^{(e)}(x, y, z, t)}{\partial t}\right]^{2} \mathrm{~d} x \mathrm{~d} y \mathrm{~d} z \\
=\frac{1}{2}\left[\mathbf{D}^{(e)}\right]^{T} \iiint\left[\mathbf{T}_{\Omega}^{(e)}\right]^{T} \rho \mathbf{T}^{(e)} \mathrm{d} x \mathrm{~d} y \mathrm{~d} z \cdot \mathbf{D}^{(e)} .
\end{array}
$$

Through the Lagrange equation of the second kind, the differential equation of motion is:

$$
\frac{\mathrm{d}}{\mathrm{d} t}\left(\frac{\partial \mathbf{W}}{\partial \dot{\mathbf{D}}}\right)-\frac{\partial \mathbf{W}}{\partial \mathbf{D}}+\frac{\partial \mathbf{V}}{\partial \mathbf{D}}=\mathbf{F} .
$$

The viscous damping theory is adopted to take into account the damping influence, and the dynamic equation of manifold element is:

$$
\mathbf{M}_{e} \ddot{\mathbf{D}}^{(e)}+\mathbf{C}_{e} \dot{\mathbf{D}}^{(e)}+\mathbf{K}_{e} \mathbf{D}^{(e)}=\mathbf{F}_{e},
$$

where $\mathbf{M}_{e}, \mathbf{C}_{e}$ and $\mathbf{K}_{e}$ are the element mass matrix, the element damping matrix and the element stiffness matrix, respectively. They are expressed as:

$$
\left\{\begin{array}{l}
\mathbf{M}_{e}=\iiint_{\Omega}\left[\mathbf{T}^{(e)}\right]^{T} \rho \mathbf{T}^{(e)} \mathrm{d} x \mathrm{~d} y \mathrm{~d} z \\
\mathbf{C}_{e}=\iiint_{\Omega}\left[\mathbf{T}^{(e)}\right]^{T} c \mathbf{T}^{(e)} \mathrm{d} x \mathrm{~d} y \mathrm{~d} z . \\
\mathbf{K}_{e}=\iiint_{\Omega}\left[\mathbf{B}^{(e)}\right]^{T} \mathbf{E B}^{(e)} \mathrm{d} x \mathrm{~d} y \mathrm{~d} z
\end{array}\right.
$$

To acquire the final global dynamic equation expediently, a transformation matrix is defined as $\mathbf{C}^{\prime(e)}$. The global generalized mass matrix $\mathbf{M}$, the global generalized damping matrix $\mathbf{C}$, and the global generalized stiffness matrix $\mathbf{K}$ are expressed as:

$$
\left\{\begin{array}{l}
\mathbf{M}=\sum_{e=1}^{n}\left[\mathbf{C}^{(e)}\right]^{T} \mathbf{M}_{e} \mathbf{C}^{(e)} \\
\mathbf{C}=\sum_{e=1}^{n}\left[\mathbf{C}^{(e)}\right]^{T} \mathbf{C}_{e} \mathbf{C}^{(e)} \\
\mathbf{K}=\sum_{e=1}^{n}\left[\mathbf{C}^{(e)}\right]^{T} \mathbf{K}_{e} \mathbf{C}^{((e)}
\end{array} .\right.
$$

The global dynamic equation of manifold element can be obtained as follows:

$$
\mathbf{M} \ddot{\mathbf{D}}+\mathbf{C} \dot{\mathbf{D}}+\mathbf{K D}=\mathbf{F} .
$$

\section{ANALYTICAL SOLUTION OF EQUATION}

\subsection{Initialization and Calculation Method of Manifold Element Equation}

It is notable that $\mathbf{D}, \dot{\mathbf{D}}$ and $\ddot{\mathbf{D}}$ in the dynamics equation of manifold element are generalized unknowns and no longer correspond to the displacement, velocity, and acceleration in the solution domain. In the NMM, the nodes of the manifold element only reflect the cover information and irrelevant to the actual physical nodes. As a result, the corresponding initial values $\mathbf{D}_{0}, \dot{\mathbf{D}}_{0}$ and $\ddot{\mathbf{D}}_{0}$ must be derived by transformation. The total number of manifold elements is $N_{x}^{\prime} \times N_{y}^{\prime} \times N_{z}^{\prime}$, where $N_{x}^{\prime}, N_{y}^{\prime}$ and $N_{z}^{\prime}$ are the number of discrete interpolation elements in $x, y$ and $z$ directions, respectively. The final calculation nodes are: 


$$
\begin{aligned}
& x_{1-k_{1}} \leq x_{2-k_{1}} \leq \cdots \leq x_{0} \leq \cdots \leq x_{N_{x}^{\prime}+k^{\prime}+1} \\
& y_{1-k_{2}} \leq y_{2-k_{2}} \leq \cdots \leq y_{0} \leq \cdots \leq y_{N_{y}^{\prime}+k^{\prime}+1} . \\
& z_{1-k_{3}} \leq z_{2-k_{3}} \leq \cdots \leq z_{0} \leq \cdots \leq z_{N_{z}^{\prime}+k^{\prime}+1}
\end{aligned}
$$

$k^{\prime}$ nodes are defined out of the physical cover to cover the entire solution domain. These nodes can help to clarify all the weight functions and meanwhile ensure that the interpolation functions near the node satisfy the coordination requirements. The initial displacement and the initial velocity of the given node are $\mathbf{U}_{0}=\mathbf{U}\left(x_{i}, y_{j}, z_{l}\right)$ and $\dot{\mathbf{U}}_{0}=\dot{\mathbf{U}}\left(x_{i}, y_{j}, z_{l}\right) . \mathbf{D}_{0}$ and $\dot{\mathbf{D}}_{0}$ can be obtained as follows:

$$
\begin{aligned}
& \mathbf{D}_{0}=\mathbf{T}_{0}^{-1} \mathbf{U}_{0}, \\
& \dot{\mathbf{D}}_{0}=\mathbf{T}_{0}^{-1} \dot{\mathbf{U}}_{0} .
\end{aligned}
$$

where

$$
\begin{aligned}
& \mathbf{T}=\left[\begin{array}{c}
\mathbf{T}_{I_{1}, J_{1}, L_{1}} \mathbf{G}_{I_{1}, J_{1}, L_{1}} \\
\vdots \\
\mathbf{T}_{I_{N_{x}^{\prime}}, J_{N_{y}^{\prime}}, L_{N_{z}^{\prime}}} \mathbf{G}_{I_{N_{x}^{\prime}}, J_{N_{t}^{\prime}}, L_{N_{z}^{\prime}}}
\end{array}\right]_{N_{x}^{\prime} \times N_{y}^{\prime} \times N_{z}^{\prime}}
\end{aligned}
$$

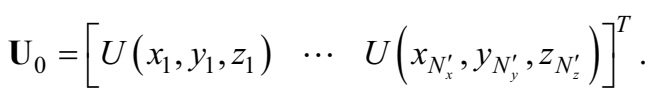

In Eq. (18), $\mathbf{G}_{I_{i} J_{i}, L_{l}}$ is the transformation matrix. And through Eq. (15), $\dot{\mathbf{D}}_{0}$ is attained as follows:

$$
\ddot{\mathbf{D}}_{0}=\mathbf{M}^{-1}\left(\mathbf{F}-\mathbf{K D}_{0}-\mathbf{C}_{0}\right) \text {. }
$$

It is worth noting that the matrices in the dynamic equations remain symmetric and positive, so the time integral method can be used for calculation. Considering the great increase of freedom degrees in the manifold elements, the Newmark method with good numerical dissipation is adopted. According to Eq. (15), the dynamic equation of manifold element at time $t+\Delta t$ can be written as

$$
\mathbf{M} \ddot{\mathbf{D}}_{t+\Delta t}+\mathbf{C D}_{t+\Delta t}+\mathbf{K D}_{t+\Delta t}=\mathbf{F}_{t+\Delta t} .
$$

The basic formulas are:

$$
\begin{gathered}
\dot{\mathbf{D}}_{t+\Delta t}=\dot{\mathbf{D}}_{t}+\left[\left(1-\gamma^{\prime}\right) \ddot{\mathbf{D}}_{t}+\gamma \ddot{\mathbf{D}}_{t+\Delta t}\right] \Delta t, \\
\mathbf{D}_{t+\Delta t}=\mathbf{D}_{t}+\mathbf{D}_{t} \Delta t+\left[\left(\frac{1}{2}-\beta^{\prime}\right) \ddot{\mathbf{D}}_{t}+\beta \ddot{\mathbf{D}}_{t+\Delta t}\right] \Delta t^{2} .
\end{gathered}
$$

When the conditions $\gamma^{\prime} \geq 0.5$ and $\beta^{\prime} \geq\left(0.5+\gamma^{\prime}\right)^{2} / 4$ are satisfied, the algorithm is stable with good numerical dissipation unconditionally, and the dynamic response function of the line teeth can be obtained.

\subsection{Natural Frequency and Mode of the Line Tooth}

In Fig. 2b, $F$ represents the meshing load on the line tooth. The meshing load $F$ can be converted into equivalent load $f$ on the constant section microelement of the curve length $\mathrm{d} s$. It is assumed that the torsion deformation and shear deformation of the line tooth micro-element are ignored. Without considering the central axial stress of the line tooth, the microelement is subjected to the equivalent external force $\mathbf{f}$, the shear force $\mathbf{q}$, the bending moment $\mathbf{m}^{\prime}$ and the inertial force $\rho A \mathrm{~d} s \cdot \partial \mathbf{u}^{2}(s, t) / \partial t^{2}$. The force analysis is shown in Fig. 4.

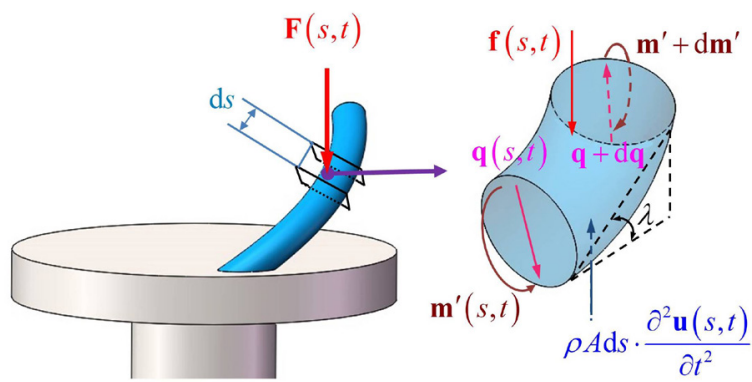

Fig. 4. Force analysis of the micro-element at any position of the line tooth

The force balance equation and the moment balance equation are established as follows:

$$
\left\{\begin{array}{c}
\rho A \mathrm{~d} s \frac{\partial^{2} \mathbf{u}(s, t)}{\partial t^{2}}+\frac{\partial \mathbf{q}(s, t)}{\partial s} d s \cdot \cos \lambda-\mathbf{f}(s, t)=0 \\
\frac{\partial m^{\prime}(s, t)}{\partial s} \mathrm{~d} s-q(s, t) \cdot \mathrm{d} s \\
+f(s, t) \cos \lambda \frac{\mathrm{d} s}{2}-\rho A \frac{\partial^{2} u(s, t)(s, t)}{\partial t^{2}} \cos \lambda \frac{\mathrm{d} s^{2}}{2}=0 \\
\mathbf{u}(s, t)=G(s) \cdot H(t) \cdot \mathbf{h}(t)
\end{array}\right.
$$

where $\mathbf{u}(s, t), G(s)$ and $\lambda$ are the displacement vector function, the mode function, and the helical angle, respectively, and $\mathbf{h}(t)$ is the unit vector.

The second-order component of the moment equation is omitted, and the motion differential equation of the cylindrical helical line tooth for the undamped free vibration is obtained as follows:

$$
\rho A \frac{\partial^{2} u(s, t)}{\partial t^{2}}+E J \frac{\partial^{4} u(s, t)}{\partial t^{4}} \cos \lambda=0
$$

Through the separated variable method, Eqs. (26) and (27) can be attained,

$$
\frac{\mathrm{d}^{2} H(t)}{\mathrm{d} t^{2}}+\omega^{2} H(t)=0
$$




$$
\frac{\mathrm{d}^{4} G(s)}{\mathrm{d} s^{4}}-\beta^{4} G(s)=0,
$$

where

$$
\beta^{4}=\frac{\omega^{2} \rho A}{E J \cos \lambda} .
$$

The solution of the Eq. (26) is:

$$
H(t)=C_{H} \sin (\omega t+\varphi),
$$

where $C_{H}$ and $\varphi$ are undetermined coefficients determined by initial conditions.

Supposing that $G(s)$ is $e^{a s}$, and the solution of the Eq. (27) is:

$$
\begin{aligned}
G(s)= & O_{1} \sin \beta s+O_{2} \cos \beta s \\
& +O_{3} \sinh \beta s+O_{4} \cosh \beta s .
\end{aligned}
$$

The boundary conditions are that one end of the line tooth is fixed and the other is free. That means:

$$
\begin{gathered}
G(s)=0 \quad \frac{\mathrm{d} G(s)}{\mathrm{d} s}=0 \quad(s=0), \\
E J \frac{\mathrm{d}^{2} G(s)}{\mathrm{d} s^{2}}=0 \quad E J \frac{\mathrm{d}^{3} G(s)}{\mathrm{d} s^{3}}=0 \quad\left(s=s_{t}\right) .
\end{gathered}
$$

Substituting the boundary conditions into Eq. (30), the frequency equation is:

$$
\cos \beta s_{t} \cdot \cosh \beta s_{t}+1=0 .
$$

Thus, the natural frequencies of each order are obtained as follows:

$$
\omega_{i}=\left(\frac{\beta_{i} s_{t}}{s}\right)^{2} \sqrt{\frac{E J \cos \lambda}{\rho A}},
$$

where $\beta_{1} s_{t}=1.875, \beta_{i} s_{t}=0.5(2 i-1),(i=2,3, \ldots, n)$, and

$$
s=\int_{t_{1}}^{t_{2}} \sqrt{x^{\prime}(t)+y^{\prime}(t)+z^{\prime}(t)} \mathrm{d} t .
$$

The mode functions corresponding to each order of natural frequency are:

$$
\begin{aligned}
G_{i}(s)= & \xi_{i}\left(\sin \beta_{i} s_{t}-\sinh \beta_{i} s_{t}\right) \\
& +\cos \beta_{i} s_{t}-\cosh \beta_{i} s_{t},
\end{aligned}
$$

where

$$
\xi_{i}=\frac{\cos \beta_{i} s_{t}+\cosh \beta_{i} s_{t}}{\sin \beta_{i} s_{t}+\sinh \beta_{i} s_{t}}, \quad(i=1,2, \ldots) .
$$

\section{VIBRATION ANALYSIS OF THE LINE TEETH}

\subsection{Meshing Excitation Analysis of Line Gear}

The dynamic excitation of line gear pair in meshing transmission can be divided into three types: meshing stiffness excitation, error excitation, and meshing impact excitation.

(1) Meshing stiffness excitation

The contact ratio of line gear in the meshing process is a periodic function. The change in the number of meshing gear pairs will cause the change in the gear angular velocity. Due to the inhomogeneity of the velocity, the vibration of the line teeth is generated. When the contact ratio of line gears changes periodically during the meshing process, the meshing stiffness of gear pairs alters in the same period. The product of the meshing stiffness $k_{s}$ of the gear pair and the relative displacement $\Delta s_{t}^{\prime}$ of the meshing line teeth in the normal direction is defined as the meshing stiffness excitation force, $F_{k}(t)$.

$$
\begin{gathered}
F_{k}(t)=k_{s} \cdot \Delta s_{t}^{\prime}, \\
k_{s}=\left[k_{m}+k_{v} \cdot \sin \left(\omega_{m} t+\varphi_{k}\right)\right], \\
k_{m}=k_{1}^{\prime} k_{2}^{\prime} /\left(k_{1}^{\prime}+k_{2}^{\prime}\right), \\
k_{v}=k_{m}(\varepsilon-1), \\
\Delta s_{t}^{\prime}=1-0.5\left(k_{l 1}-k_{l 2}\right),
\end{gathered}
$$

where $k_{m}, k_{1}^{\prime}$ and $k_{2}^{\prime}$ are the average stiffness of gear pairs, the single-tooth stiffness of the driving line tooth and the driven line tooth, respectively, $k_{l 1}$ and $k_{l 2}$ are the correction coefficients of the centre curve of the driving line gear and the driven line gear, respectively, and $\varepsilon$ is the coincidence degree of the line gears.

\section{(2) Error excitation}

The meshing error of the line gears is caused by machining error and installation error. The machining and manufacturing methods of line gears can be divided into three types: profiling, stereo lithograph apparatus (SLA), and selective laser melting (SLM). The comparison of these three manufacturing methods is shown in Table 1.

Table 1. Comparison of manufacturing methods of the line gear [24] to [26]

\begin{tabular}{lccc}
\hline & $\begin{array}{c}\text { Shape } \\
\text { accuracy }\end{array}$ & $\begin{array}{c}\text { Dimensional } \\
\text { accuracy }[\mathrm{mm}]\end{array}$ & $\begin{array}{c}\text { Surface roughness, } \\
R a[\mu \mathrm{m}]\end{array}$ \\
\hline Profiling & poor & $<0.02$ & 5 \\
\hline SLA & good & $<0.02$ & 20 \\
\hline SLM & good & $<0.03$ & 25 \\
\hline
\end{tabular}

As shown in Table 1, error excitations of the line teeth are different, depending on the methods by which they were manufactured. The error excitation is mainly caused by the deviation of tooth shape and the surface roughness of the teeth. The deviation of 
tooth shape comes from the discrepancy between the theoretical meshing curve and the actual meshing curve and leads to the vibration during the meshing. The surface roughness of line teeth can meet the requirements by secondary machining. Therefore, the vibration caused by roughness can be ignored. The error excitation is defined as:

$$
F_{l}(t)=k_{s} \cdot\left(1-k_{c}\right),
$$

where $k_{c}$ is the correction coefficient of the meshing curve.

\section{(3) Meshing impact excitation}

Due to the size error and pitch error of the line gear, the actual meshing point of engaging-in and engaging-out usually deviate from the theoretical meshing point of engaging-in and engaging-out in the meshing process, which disturbs the rotating speed of the meshing line teeth. The vibrations at the beginning and the end of gear meshing are defined as the approach shock and recess shock, respectively.

When the line teeth fail to rotate in time into the meshing state at the meshing point of engagingin due to the pitch deviation, the approach shock will occur. Meanwhile, when the meshing line teeth fail to separate in time at the meshing point of engaging-out due to tooth shape error or pitch deviation, and the latter pair of the meshing line teeth may rotate into a meshing state at the meshing point of engaging-in. In this case, the former pair of line teeth has to change their velocity to maintain their continuous motion transition, which will cause the recess shock.

The difference between the theoretical time $T_{0}$ and the actual time $t_{0}$ at the meshing point of engaging-in is defined as the action time of the approach shock.

$$
\begin{aligned}
& \Delta t=\left|T_{0}-t_{0}\right|, \\
& T_{0}=\frac{2 \pi}{n_{1} \omega_{1}} . \\
& x_{2 c}=\cos \frac{\pi+t_{c}}{i_{12}} \cdot\left[m \cdot \cos \theta-\left(n t+n \pi+\frac{k_{m} d}{2}+\frac{d}{2 k_{m}}\right) \sin \theta+a+\frac{k_{n} d}{2} \sin \frac{t_{c}+\pi}{i_{12}}\right] \\
& \left\{y_{2 c}=\sin \frac{\pi+t_{c}}{i_{12}} \cdot\left[m \cdot \cos \theta-\left(n t+n \pi+\frac{k_{m}^{2} d+d}{2 k_{m}}+\frac{d}{2}\right) \sin \theta+a\right]+\frac{k_{n} d}{2} \cos \frac{t_{c}+\pi}{i_{12}},\right. \\
& z_{2 c}=-m \cdot \sin \theta-\left(n t_{c}+n \pi+\frac{k_{m} d}{2}+\frac{d}{2 k_{m}}\right) \\
& k_{m}=\frac{m}{\sqrt{m^{2}+n^{2}}}, \quad k_{n}=\frac{n}{\sqrt{m^{2}+n^{2}}}, \quad-\pi \leq t_{c} \leq-0.45 \pi
\end{aligned}
$$

The approach shock force at any time $t$ in the $\Delta t$ can be defined as:

$$
F_{r}=\frac{M_{d} \cdot \Delta \dot{U}_{d}}{\Delta t}
$$

where $M_{d}$ and $\Delta \dot{U}_{d}$ are the mass and the velocity variation of the single meshing line tooth.

Due to the inertia, the recess shock force will reduce to a negligible level, so only the approach shock in the meshing impact excitation needs to be considered.

\subsection{Example}

In the meshing process of the line gear, meshing stiffness excitation and error excitation are the periodic excitation and the meshing impact excitation is the transient excitation. As the dynamic response of the line teeth mainly belongs to the steady-state response, only meshing stiffness excitation and error excitation are needed to be concerned. Supposing the angular velocity $\omega_{m}$ of the driving gear is $\pi\left[\mathrm{rad} \cdot \mathrm{s}^{-1}\right.$, and the excitation function is:

$$
\begin{gathered}
F(t)=F_{k}(t)+F_{l}(t)=F\left[1+(\varepsilon-1) \sin \left(\omega_{m} t+\varphi_{k}\right)\right], \\
F=\frac{k_{1}^{\prime} k_{2}^{\prime}}{k_{1}^{\prime}+k_{2}^{\prime}}\left[2-0.5\left(k_{l 1}+k_{l 2}\right)-k_{c}\right], \\
\varepsilon=\Delta \varphi_{1} n_{1} /(2 \pi),
\end{gathered}
$$

where $\Delta \varphi_{1}$ and $\varphi_{k}$ are the rotation angle of the single meshing line tooth and the initial phase.

The centre curve equations of the driving line tooth are:

$$
\left\{\begin{array}{l}
x_{c_{1}}=m \cos t_{c} \\
y_{c_{1}}=m \sin t_{c}, \quad-\pi \leq t_{c} \leq-0.475 \pi . \\
z_{c_{1}}=n t_{c}+n \pi
\end{array}\right.
$$

According to the theory of line gear meshing, the centre curve equations of the driven line tooth can be 
where $i_{12}$ is the transmission ratio, $\theta$ is the included angle of the gear surfaces, $n_{1}$ is the tooth number of the driving gear, $m$ is the base circle radius of the driving gear, $n$ is the thread pitch parameter, $k_{1 d}$ is the clearance coefficient of driving line gear, $a$ is the centre distance, $d$ is the diameter of line teeth, and $n_{2}$ is the number of the driven gear.

The main parameters of the cylindrical helical driving line gear and the corresponding driven line gear are shown in Table 2 as an example.

Table 2. Main parameters of the line gears in the example

\begin{tabular}{cccccc}
\hline$i_{12}$ & $\theta\left[^{\circ}\right]$ & $n_{1}$ & $m[\mathrm{~mm}]$ & $n[\mathrm{~mm}]$ & $k_{1 d}$ \\
\hline 2.5 & 150 & 6 & 30 & 20 & 1.5 \\
\hline$k_{l 2}$ & $k_{l 1}$ & $n_{1}$ & $d[\mathrm{~mm}]$ & $a[\mathrm{~mm}]$ & $k_{c}$ \\
\hline 0.95 & 0.9 & 15 & 1.5 & 120 & 0.95 \\
\hline
\end{tabular}

The Newmark parameters $\gamma^{\prime}$ and $\beta^{\prime}$ are selected as 0.5 and 0.25 , respectively. Let the damping $c$ be zero, and the dynamic response function of the driving tooth is:

$$
\begin{aligned}
& U(x, y, z, t)= \\
& \frac{2 F}{\rho A s} \sum_{i=1}^{n} \frac{\left(\sin \alpha_{i}(x+m)+\sin \alpha_{i} y\right)+(2 i-1) \cdot \sin \alpha_{i} z}{(2 i+1) \cdot \omega_{i}^{2}\left[1-\left(\omega / \omega_{i}\right)^{2}\right]} \\
& \cdot \sum_{i=1}^{n}\left(\sin \omega t-\frac{\omega}{\omega_{i}} \sin \omega_{i} t\right),
\end{aligned}
$$

where $\alpha_{i}=(2 i-1) / 2 s$

The displacement curves of undamped forced vibration of the driving tooth in $x, y$ and $z$ directions can be drawn as follows:

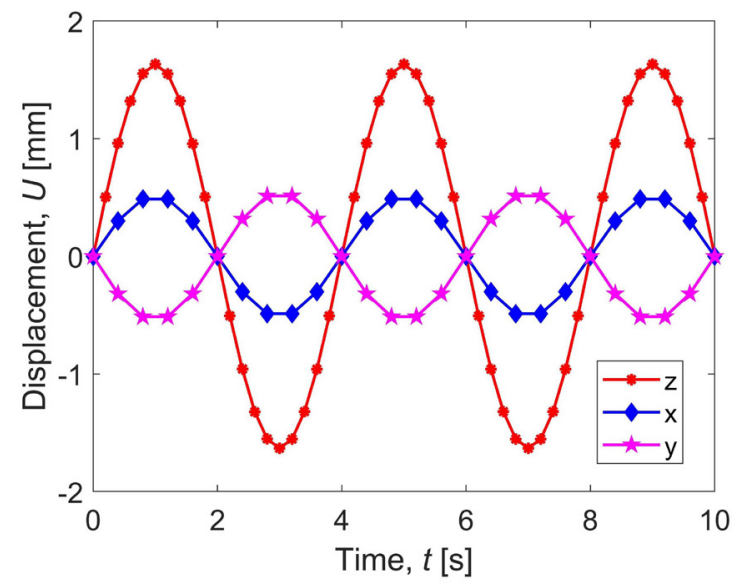

Fig. 5. Undamped forced vibration response diagram of the driving tooth in $x, y$ and $z$ directions
From Eq. (33), the natural frequency function is attained:

$$
\left\{\begin{array}{c}
\omega_{i}=\frac{d \cdot\left(\beta_{i} s_{t}\right)^{2}}{4 m^{2}\left(1+\tan ^{2} \lambda\right) \Delta t^{2}} \sqrt{\frac{E \cos \lambda}{\rho}} \\
\beta_{i} s_{t}=1.875, \beta_{i} s_{t}=\frac{2 i-1}{2} \pi,(i=2,3, \ldots, n)
\end{array},\right.
$$

where $\rho$ is the material density, $m$ is the base circle radius, $\lambda$ is the helix angle, $\Delta t$ is the line tooth length parameter, and $E$ is the elasticity modulus of the material.

Eq. (53) indicates a one-to-one correspondence mathematical relationship between the natural frequency and the design parameters of the line gears. The parameter values of the line gears have an applicable range shown in Table 3, and the function relationship is shown in Fig. 6.

Table 3. Applicable ranges of parameters affecting the natural frequency

\begin{tabular}{ccccc}
\hline$d[\mathrm{~mm}]$ & $\rho\left[\mathrm{g} \cdot \mathrm{cm}^{-3}\right]$ & $\lambda\left[^{\circ}\right]$ & $m[\mathrm{~cm}]$ & $\Delta t[-]$ \\
\hline 1 to 10 & 1 to 10 & 25 to 25 & 2 to 10 & $\pi / 3$ to $3 \pi$ \\
\hline
\end{tabular}
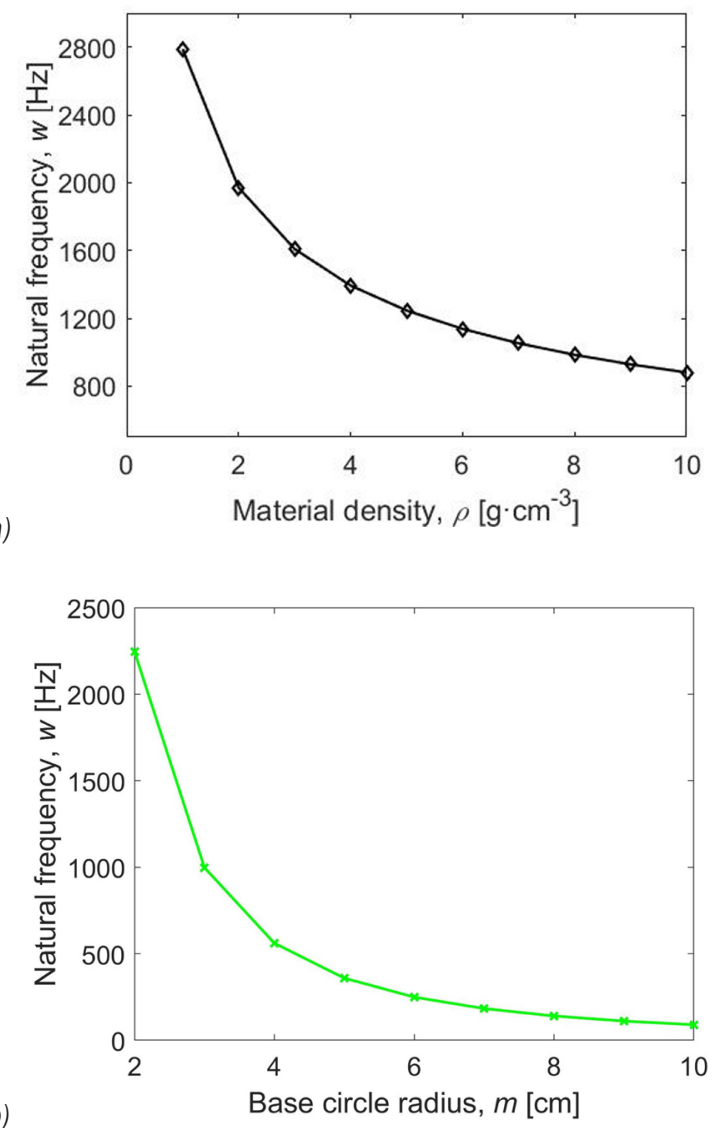

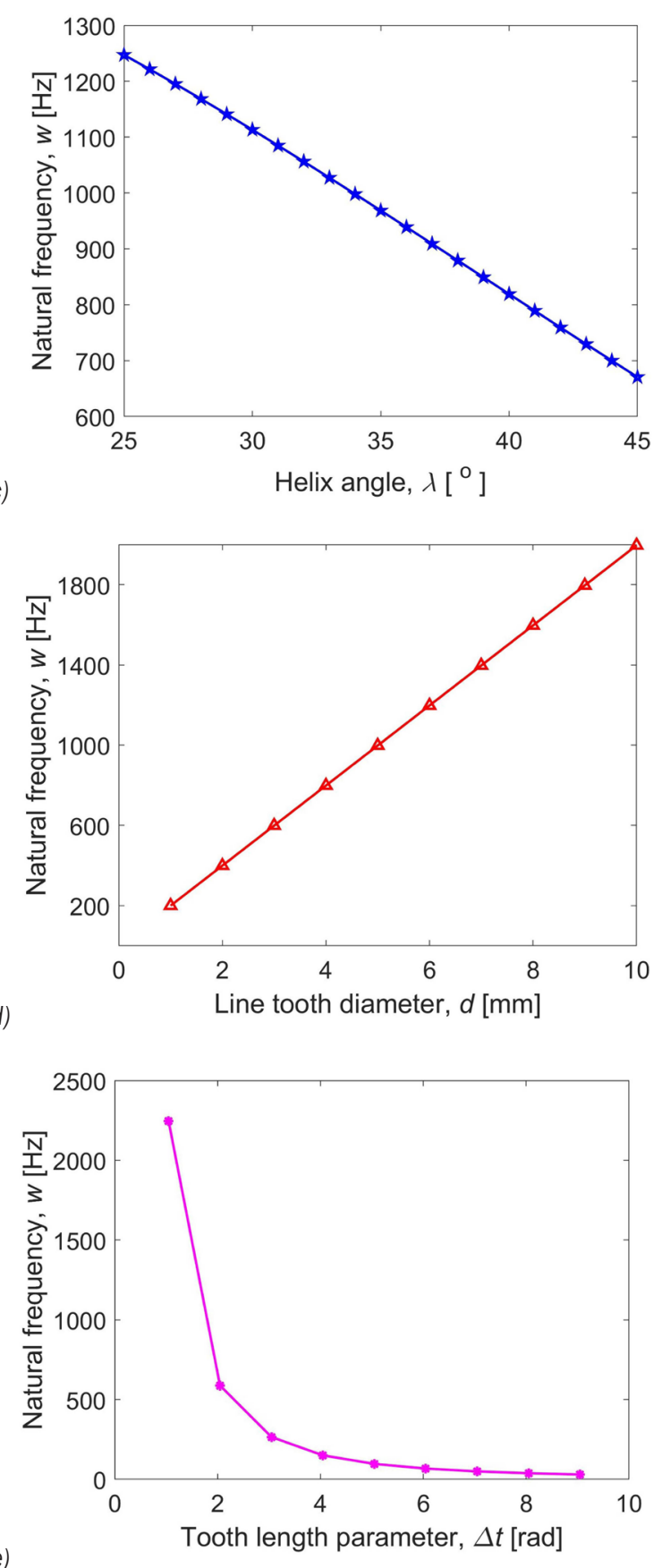

Fig. 6. Function relationship between the natural frequency and main parameters of line gears; a) material density, b) line gear base radius, c) helix angle of line tooth, d) line tooth diameter; and e) line tooth length parameter

As shown in Fig. 6, the natural frequency of the line tooth is proportional to the diameter of the line tooth but inversely proportional to the helix angle approximately. The natural frequency is strictly inversely proportional to material density, base circle radius and line tooth length.
The transmission error can be caused by various types of errors, for instance, installation error and tooth profile error. The line gear meshing stress nephogram and the transmission error curve are shown in Fig. 7. The transmission error results computed with FEM and NMM are essentially consistent. We use an aluminium alloy as the manufacturing material. The elasticity modulus $E$ of the material is $72 \mathrm{GPa}$. The material density $\rho$ is $2.8 \mathrm{~g} \cdot \mathrm{cm}^{-3}$. The Poisson's ratio $\mu$ is 0.3 . The natural mode of the line tooth can be obtained from the natural frequency. The natural modes of the first four orders and their specific descriptions are shown in Table 4.
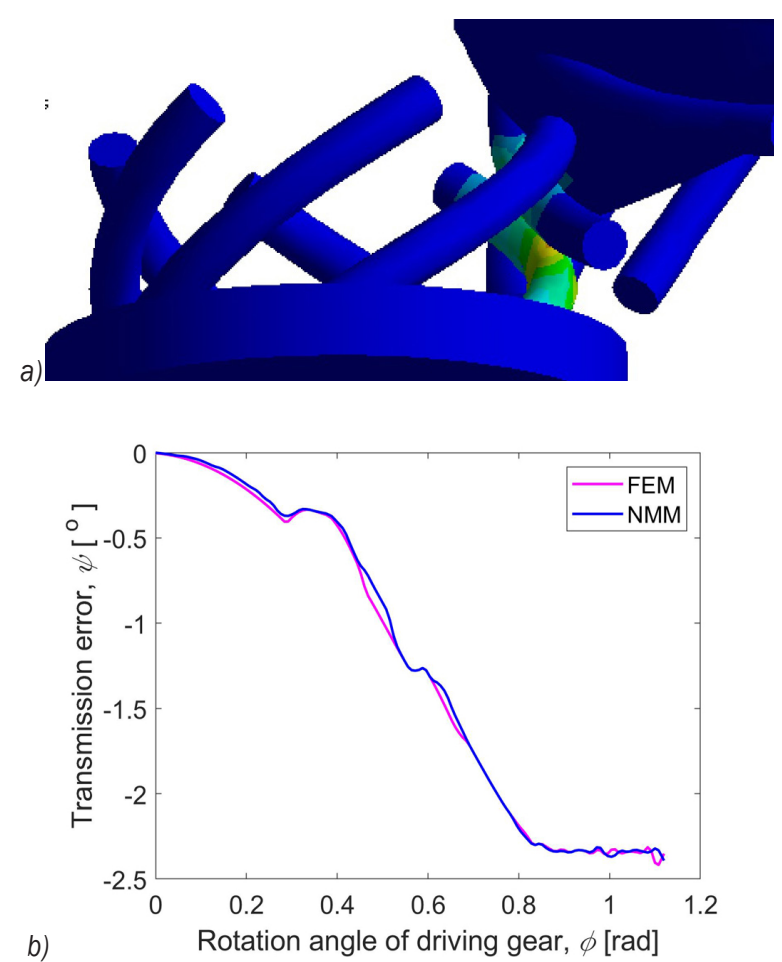

Fig. 7. Line gear transmission error analysis; a) gear meshing stress nephogram, b) transmission error curve

Fig. 5 shows that the amplitude of the line tooth in $z$ direction is larger than that in $x$ and $y$ directions. The comparison of frequency results between the FEM and NMM is shown in Table 4. The difference in the results could be caused by calculation error and grid division error. As shown in Table 4, the vibration of the line tooth mainly exists in the axial direction of the line gear and the curvature direction of line teeth. The force direction of the meshing line teeth is shown in Fig. 2. Obviously, the vibration of the line tooth in $z$ direction is equal to the vibration of the line tooth in the axial direction of the gear, and the vibration in the curvature direction of the line tooth is composed 
of the vibration in $x$ and $y$ directions. The line tooth vibration in the axial direction is more significant than that in the curvature direction. The numerical results from the example indicate that the natural vibration modes of the first four orders are coincident with the dynamic model according to NMM.

Table 4. The vibration characteristics of the first four orders of line teeth

\begin{tabular}{|c|c|c|c|c|}
\hline \multirow[t]{2}{*}{ Order } & \multicolumn{2}{|c|}{$\begin{array}{c}\text { Natural } \\
\text { frequency }[\mathrm{Hz}]\end{array}$} & \multirow[t]{2}{*}{ Corresponding mode } & \multirow[t]{2}{*}{ Descriptions } \\
\hline & NMM & FEM & & \\
\hline $\begin{array}{l}\text { The } \\
1 \text { st } \\
\text { order }\end{array}$ & 272 & 306 & & $\begin{array}{l}\text { no node vibration } \\
\text { of the line tooth } \\
\text { in the gear axial } \\
\text { direction }\end{array}$ \\
\hline $\begin{array}{l}\text { The } \\
\text { 2nd } \\
\text { order }\end{array}$ & 1704.5 & 1716.8 & & $\begin{array}{l}\text { no node vibration } \\
\text { of the line } \\
\text { tooth in the line } \\
\text { tooth curvature } \\
\text { direction }\end{array}$ \\
\hline $\begin{array}{l}\text { The } \\
\text { 3rd } \\
\text { order }\end{array}$ & 4771.9 & 4834.2 & & $\begin{array}{l}\text { single node } \\
\text { vibration of the } \\
\text { line tooth in } \\
\text { the gear axial } \\
\text { direction }\end{array}$ \\
\hline $\begin{array}{l}\text { The } \\
4^{\text {th }} \\
\text { order }\end{array}$ & 9353 & 9373.5 & & $\begin{array}{l}\text { single node } \\
\text { vibration of the } \\
\text { line tooth in } \\
\text { the line tooth } \\
\text { curvature } \\
\text { direction }\end{array}$ \\
\hline
\end{tabular}

\section{CONCLUSION}

The innovative method, NMM, is employed to investigate the dynamic characteristics and alleviate the vibration conditions of line gears. A B-spline interpolation function is used as the mathematical cover of the NMM since it can provide continuous local support and local control. The displacement function of the line teeth and the dynamic equation of the manifold element are constructed, and the dynamic response function of the line teeth is acquired. The results show that the line tooth vibration in the axial direction is more significant than that in the curvature direction. A micro-element of the line tooth with constant cross-section is used for stress analysis, and the constant cross-section vibration differential equation of the cylindrical helical cantilever is attained. Furthermore, the frequency equation and the natural frequency of the cylindrical helical line tooth are attained. Furthermore, the function relationship between the design parameters of line gears and the natural frequency is revealed. The natural frequency of the line tooth is proportional to the diameter of the line tooth but roughly inversely proportional to the helix angle of the line tooth. In addition, the natural frequency is strictly inversely proportional to material density, base circle radius and line tooth length. In the end, the comparison of frequency results between FEM and NMM and the natural modes of the first four order of the line gear verify the dynamic model according to the NMM.

\section{ACKNOWLEDGMENTS}

The authors gratefully acknowledge the generous joint support provided by National Natural Science Foundation of China (Grant No. 51905113), Guangxi Natural Science Foundation (Grant No. 2017GXNSFBA198233), Guangxi Key Laboratory of Manufacturing System \& Advanced Manufacturing Technology (Grant No. 16-380-12S002), and Innovation Project of Guangxi Graduate Education (Grant No. YCSW2020018).

\section{NOMENCLATURE}

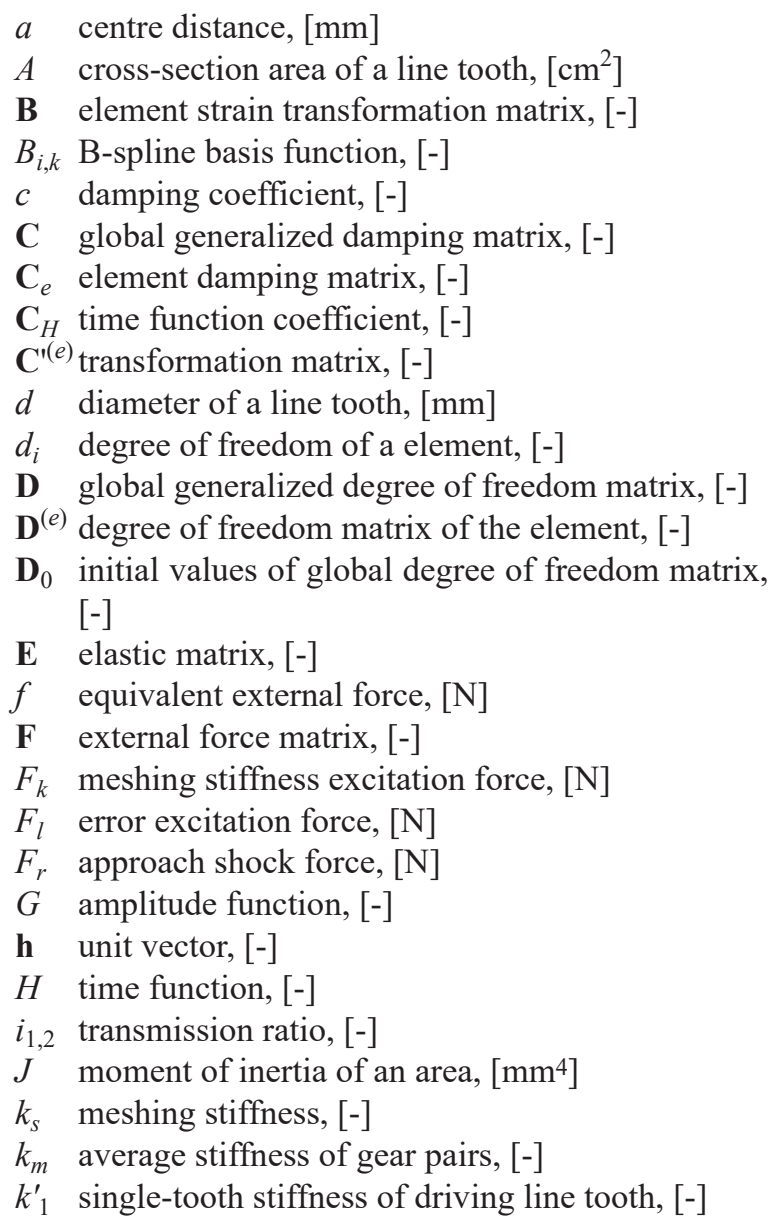


$k_{2}^{\prime} \quad$ single-tooth stiffness of driven line tooth, [-]

$k_{l 1}$ correction coefficients of the centre curve of driving line tooth, [-]

$k_{l 2}$ correction coefficients of the centre curve of driven line tooth, [-]

$k_{c} \quad$ correction coefficient of the meshing curve, [-]

$k_{d} \quad$ clearance coefficient of driving line gear, [-]

$\mathbf{K}_{e}$ element stiffness matrix, [-]

K global generalized stiffness matrix, [-]

$m$ base circle radius of the driving gear, [mm]

$m^{\prime}$ bending moment, $[\mathrm{N} \cdot \mathrm{m}]$

$\mathbf{M}_{e}$ element mass matrix, [-]

M global generalized mass matrix, [-]

$n$ thread pitch parameter, $[\mathrm{mm}]$

$n_{1}$ tooth number of the driving gear, [-]

$n_{2}$ tooth number of the driven gear, [-]

$\mathbf{N}_{e}$ interpolation function matrix of an element, [-]

$q$ shear force, $[\mathrm{N}]$

Q differential operator matrix, [-]

$s \quad$ curve length, [mm]

S order matrix of the local cover function, [-]

$t$ time,[s]

$\mathbf{T}^{(e)}$ covering matrix of an element, [-]

$u$ displacement of the micro-element, [mm]

U global generalized displacement matrix, [mm]

$\mathbf{U}^{(e)}$ displacement matrix of an element, [mm]

$\mathbf{V}^{e}$ strain energy matrix, [-]

$\mathbf{W}^{e}$ kinetic energy matrix, [-]

$\beta$, Newmark parameter, [-]

$\gamma^{\prime} \quad$ Newmark parameter, [-]

$\boldsymbol{\varepsilon}^{e} \quad$ strain matrix of an element, [-]

$\theta$ angle of central axis, $\left[{ }^{\circ}\right]$

$\lambda$ helix angle, $\left[{ }^{\circ}\right]$

$\rho$ material density, $\left[\mathrm{g} \cdot \mathrm{cm}^{-3}\right]$

$\boldsymbol{\sigma}^{e} \quad$ stress matrix of an element, [-]

$\varphi \quad$ angle of initial phase, [rad]

$\phi \quad$ rotation angle of driving gear, $[\mathrm{rad}]$

$\psi \quad$ transmission error, $\left[{ }^{\circ}\right]$

$\omega$ excitation frequency, $[\mathrm{Hz}]$

\section{REFERENCES}

[1] Zheng, F., Han, X., Lin, H., Zhang, M., Zhang, W. (2018). Design and manufacture of new type of non-circular cylindrical gear generated by face-milling method. Mechanism and Machine Theory, vol. 122, p. 326-346, D0l:10.1016/j. mechmachtheory.2018.01.007.

[2] Chen, Y., Lv, Y., Ding, J., Chen, Z. (2013). Fundamental design equations for space curve meshing skew gear mechanism. Mechanism and Machine Theory, vol. 70, p. 175-188, D0I:10.1016/j.mechmachtheory.2013.07.004.

[3] Hai, H., Yin, Y. Q., De Xin, T., Hong, X. L. (2013). Multi-objective optimization design of hard gear-face point-line meshing gear.
Applied Mechanics and Materials, vol. 271-272, p. 10271031, D0I:10.4028/www.scientific.net/AMM.271-272.1027.

[4] Chen, Y., Huang, H., Lv, Y. (2016). A variable-ratio line gear mechanism. Mechanism and Machine Theory, vol. 98, p. 151163, D0I:10.1016/j.mechmachtheory.2015.12.005.

[5] Chen, Y., Liang, S., Ding, J. (2014). The equal bending strength design of space curve meshing wheel. Journal of Mechanical Design, vol. 136, no. 6, art. ID 061001, DOI:10.1115/1.4027160.

[6] Yi, Y., Liyan, C., Hang, L., Yiping, D. (2019). Non-linear dynamic response of a spur gear pair based on the modeling of periodic mesh stiffness and static transmission error. Applied Mathematical Modelling, vol. 72, p. 444-469, Dol:10.1016/j. apm.2019.03.026.

[7] Meng, Z., Shi, G., Wang, F. (2020). Vibration response and fault characteristics analysis of gear based on time-varying mesh stiffness. Mechanism and Machine Theory, vol. 148, art. ID 103786, D0I:10.1016/j.mechmachtheory.2020.103786.

[8] Belingardi, G., Cuffaro, V., Cura, F. (2018). Multibody approach for the dynamic analysis of gears transmission for an electric vehicle. Proceedings of the Institution of Mechanical Engineers, Part C: Journal of Mechanical Engineering Science, vol. 232, no. 1, p. 57-65, Dol:10.1177/0954406216674981.

[9] Cirelli, M., Giannini, O., Valentini, P.P. Pennestri, E. (2020). Influence of tip relief in spur gears dynamic using multibody models with movable teeth. Mechanism and Machine Theory, vol. 152, art. ID 103948, Dol:10.1016/j. mechmachtheory.2020.103948.

[10] Cirelli, M., Valentini, P.P., Pennestri, E. (2019). A study of the non-linear dynamic response of spur gear using a multibody contact based model with flexible teeth. Journal of Sound and Vibration, vol. 445, p. 148-167, Dol:10.1016/j. jsv.2019.01.019.

[11] Ebrahimi, S., Eberhard, P. (2006). Rigid-elastic modeling of meshing gear wheels in multibody systems. Multibody System Dynamics, vol. 16, p. 55-71, Dol:10.1007/s11044-006-90217.

[12] Cooley, C.G., Liu, C., Dai, X., Parker, R.G. (2016). Gear tooth mesh stiffness: A comparison of calculation approaches. Mechanism and Machine Theory, vol. 105, p. 540-553, D0I:10.1016/j.mechmachtheory.2016.07.021.

[13] Luo, Y., Baddour, N., Liang, M. (2019). A shape-independent approach to modelling gear tooth spalls for time varying mesh stiffness evaluation of a spur gear pair. Mechanical Systems and Signal Processing, vol. 120, p. 836-852, Dol:10.1016/j. ymssp.2018.11.008.

[14] Wang, X., Li, S. (2016). Free vibration analysis of functionally graded material beams based on Levinson beam theory. Applied Mathematics and Mechanics-English Edition, vol. 37, p. 861-878, DOI:10.1007/s10483-016-2094-9.

[15] Zhang, J., Ge, R., Zhang, L. (2019). Transverse free vibration analysis of a tapered Timoshenko beam on visco-Pasternak foundations using the interpolating matrix method. Earthquake Engineering and Engineering Vibration, vol. 18, p. 567-578, D0l:10.1007/s11803-019-0522-9.

[16] Zhao, G., Wu, Z. (2017). Coupling vibration analysis of rotating three-dimensional cantilever beam. Computers \& Structures, vol. 179, p. 64-74, DOl:10.1016/j.compstruc.2016.10.024. 
[17] Zhou, D., Fang, J., Wang, H., Zhang, X. (2019). Threedimensional dynamics analysis of rotating functionally gradient beams based on Timoshenko beam theory. International Journal of Applied Mechanics, vol. 11, no. 4, art ID 19500404, D0I:10.1142/S1758825119500406.

[18] Yang, Y., Sun, G., Cai, K., Zheng, H. (2018). A high order numerical manifold method and its application to linear elastic continuous and fracture problems. Science China Technological Sciences, vol. 61, p. 346-358, D0l:10.1007/ s11431-016-9070-8.

[19] Yu, C., Liu, F., Xu, Y. (2018). An h-adaptive numerical manifold method for solid mechanics problems. Science China Technological Sciences, vol. 61, p. 923-933, D0l:10.1007/ s11431-017-9143-9.

[20] Yang, Y., Sun, G., Zheng, H. (2020). A high-order numerical manifold method with continuous stress/strain field. Applied Mathematical Modelling, vol. 78, p. 576-600, D0l:10.1016/j. apm.2019.09.034.

[21] Zhang, Z., Zhang, X., Lue, W. (2010). Numerical method based on compatible manifold element for thin plate bending. Chinese Journal of Mechanical Engineering, vol. 23, no. 1, p. 100-109, DOI:10.3901/CJME.2010.01.100.

[22] Wen, W., Jian, K., Luo, S. (2013). 2D numerical manifold method based on quartic uniform B-spline interpolation and its application in thin plate bending. Applied Mathematics and Mechanics-English Edition, vol. 34, p. 1017-1030, DOI:10.1007/s10483-013-1724-x.

[23] Vadlamani, S., Arun, C.0. (2019). Construction of beam elements considering von Karman non-linear strains using B-spline wavelet on the interval. Applied Mathematical Modelling, vol. 68, p. 675-695, D0l:10.1016/j. apm.2018.11.042.

[24] Chen, Y., Sun, L., Wang, D., Yang, Y., Ding, J. (2010). Investigation into the process of selective laser melting rapid prototyping manufacturing for space-curve-meshingwheel. Advanced Materials Research, vol. 135, p. 122-127, DOI:10.4028/www.scientific.net/AMR.135.122.

[25] Kruth, J.P., Froyen, L., Van Vaerenbergh, J., Mercelis, P., Rombouts, M., Lauwers, B. (2004). Selective laser melting of iron-based powder. Journal of Materials Processing Technology, vol. 149, no. 1-3, p. 616-622, D0l:10.1016/j. jmatprotec.2003.11.051.

[26] Wu, W., Yang, Y., Lai, K. (2007). Process analysis of rapid prototyping with selective laser melting. Journal of South China University of Technology Natural Science Edition vol. 35, p. 22-27, D0l:10.1002/jrs.1570. (in Chinese) 